\title{
The Effect of Gas Energy Propagation Velocity on the Transport Security
}

\author{
Song Sun ${ }^{1, a^{*}}$ \\ ${ }^{1}$.State Key Laboratory of Disaster Prevention and Mitigation of Explosive and Impact,PLA University \\ of Science and Technology,Nanjing,210007,China; \\ asunsongky23@163.com
}

Keywords: gas energy transport; flame velocity;combustion;energy security

Abstract: With the development of technology and the progress of life,gas energy play a more important role in our daily use. The transmission process is a key part of the security use of energy.

In the transport process, the flame velocity caused by the combustion of enengy have a crucial impact on the pressure and safety performance.This article taking methane as an example,analyzing the pressure and safety performance in the process of transport from the aspects of energy burning and flame velocity. Theoretical study of premixed flame propagation in vessels is described.The study provides understanding of flame propagation and the acceleration of flame and also introduce a method in velocity assessment through a mathematical model.The influence factor and the development tendency of flame transport velocity are also described in the artical.

\section{Introduction}

With the development of economy and more attention paid on environment issues, the transmission of gas energy through pipeline gradually get widespread use. Now it has played an irreplaceable role in our daily life.But with the lagre-scale use of gas energy, explosion accident of gas in pipeline-transmission happened with a higher frequencies. The security of gas energy during transmission has become a serious problem we have to face.

In our real life,methane is tha main component of natural gas.After it has been ignited,the flame propagation velocity has a direct impact on the pressure and security in the pipeline.Now,through the factors which influence the process of flame propagation we can analysis the flame burning velocity. Lay the foundation for further assess of the gas transportation safety and reduce the occurrence of gas explosion accidents.

\section{Flame profile in the process of flame propagation}

The combustion of gaseous fuel occurs in the pipeline is generally in the form of deflagration. Its propagation velocity is usually attributed to the result of joint action of the combustion speed and propagation speed. In the process of the combustion propagation, gas concentration, the scale of tube,the inner wall of the pipe and many other factors has a direct impact on the propagation velocity and the shape of flame.In the ideal condition,flame pront in pipeline is a regular plane.But in the actural process of propagation,this situation is difficult to appear because of the flame distortion effects.For the changes of flame front in propagation,predecessors have done lots of observation and research through optical instruments.Martstein[1]found that flame propagation has cellular structure in the glass pipe.Searby[2] also observed such phenomena through experiment.He analyzed that unstable disturbances of flame front in the process of flame propagation is the reason for this phenomenon.Hereafter,Lee[3] observed that mushroom flames and tulip flames will also appear in the process of flame combustion propagation.

Analysis the reason for the diversification of flame front in the process of propagation,it's considered that while in the initial phase of combustion flame diffuses in spherical form.But in the process of diffusion flame front will be floded because of self-turbulence. The part of unreacted portion contact with air got expanded and flame combustion velocity got accelerated .The speed of middle section is faster than both sides and form the shape of mushroom flames.If the inner wall of tube is rough,it will produce the viscous effect and make a shear plane as well as velocity gradient in the 
process of flame propagation.Gas which disturbed due to shearing action aggravate the turbulence of flow field and make the flame near the pipe wall faster than middle section. Thus tulip flames got formed.

\section{The factors that influence the flame propagation velocity}

Gas explosion in pipeline is usually divided into slow-burning,deflagration and detonation.The velocity of flame propagation is quite slow in the phase of slow-burning and mainly embodied in the process of combustion and diffusion in chemical reaction of flame and unburned gas.Deflagration is the most common form of gas combustion in tube. In the process of deflagration there will produce a wave front and flame front. The faster wave front and the flame front behind will form three-zone structure with two sides[4].For the prevention to explosion disaster of gas combustion typically start from the stage of deflagration to reduce the threat. When the flame propagation get accelerated and catch up with the wave front,deflagration will transport into detonation.In the phase of detonation, a sharp rise of pressure generated in the process of flame propagation and velocity can reach thousands of meters per second.Great damage will occur because of detonation so it's essential to avoid DDT in energy transportion.

\section{Effect of gas concentration on flame propagation velocity}

Flame propagation speed is usually decided jointly by burning velocity and propagation velocity.The rate of combustion is the reaction rate with unburned gas which refers to a perpendicular to the burning flame front. It's main decided by the nature gas itself and directly determined by the type and concentration of gas. The concentration of gas directly affects the reaction rate and the resulting pressure,so we must understand that:(1)the explosion limit:the limit of gas explosion occurred in air,explosion won't happen when concentration is higher or lower than the limit no matter how high the ignition energy given;(2)Stoichiometric concentration:under this concentration gas get completely reaction and generally considered that the explosion intensity is the greaster in this condition.(3)Optimal concentration:it is generally believed that under this concentration the rate of combustion reaches maximum.In general, optimal concentration is higher than stoichiometric concentration due to incomplete reaction.So in experimental research we generally take stoichiometric concentration as the most dramatic concentration.

According to the types of gas,gas energy can be divided into low reactivity, the reaction activity and high reactivity.Methane which makes up a large propation of new gas energy is low reactivity and in experimental studies methane-air mixture usually used as research object.The limit of methane explosion is $5.3 \%$ to $14 \%$ and the stoichiometric mixtures of methane is $9.5 \%$.Gao[5] obtained the reaction temperature and pressure in different concentrations after the reaction through series of experiment and shown in Table1. Through the experimental analysis we can get that the combustion reaction mechanism will be changed dramatically under different reaction concentration and the velocity and pressure of combustion products have a huge difference.Under stoichiometric concentration methane-air mixture react dramatically and reaches the highest temperature and pressure. Therefore in the process of gas energy transport,we can avoid gas explosion by keeping gas concentration out of the explosion limit,adding inert gas,eliminating sources of ignition or some other measures.

Table 1 The temperature and pressure after explosion

\begin{tabular}{c|c|c|c|c}
\hline fuel & concentration & wavelength[mm] & temperature[K] & pressure[atm] \\
\hline \multirow{2}{*}{$\mathrm{CO}$} & $74 \%$ & 650 & 1139 & 3.66 \\
\cline { 2 - 5 } & $12.5 \%$ & 500 & 1171 & 3.51 \\
\hline \multirow{2}{*}{$\mathrm{CH}_{4}$} & $5.3 \%$ & 500 & 1232 & 4.13 \\
\cline { 2 - 5 } & $9.5 \%$ & 500 & 1851 & 6.09 \\
\hline
\end{tabular}




\section{Effect of flame stretch and instabilities on flame propagation velocity}

At the initial stage of ignition,the concentraton in the region around ignition is basically equal. The flame front is spherical and flame front in the pipe presents a regular plane theoretically.But in the actual process, the farther away from the explosion point, the more irregular flame front is. This is due to the instability of the flame itself in the process of transmission.In the burning front the flame front can be divided into a series of small explosions source type,producing eddy which have a great influence on the flame front and make the surface expand rapidly as well as intensify the energy release.At the initial stage of combustion,the irregular distribution of temperature and density and other causal factors will all cause turbulence on flame front,make the flame stretched and extended. The root of the flame instability is the inevitable imbalance of chemical reactions and the fuel flows.So this phenomenon is called self-eddy in explosion.

Rota[6] developed a similar simplified model for combustion and describe the self-stability by $\lambda_{0}$. The influence parameters of flame growth $\varphi_{1}$ and the parameters of flame transmission $\varphi_{2}$ was put forward to assessment the instability of flame. They evaluated three constants $\theta, \beta_{1}$ and $\beta_{2}$, to minimize the error between their model and experiment data.They set a value of 4000 for the cellular flame Reynolds number and fitting the following equation to describe the self-instability of flame combustion.

$\lambda_{0}=\varphi_{1} \varphi_{2}$

$\boldsymbol{\varphi}_{1}=\left\{\begin{array}{l}1, \text { if } \operatorname{Re}_{f}<4000 \\ \frac{\operatorname{Re}_{f}}{4000}, \text { otherwise }\end{array}\right\}$

$\boldsymbol{\varphi}_{2}=\max \left\{1, \beta_{1}\left(\frac{\operatorname{Re}_{V}}{10^{6}}\right)^{\frac{\beta_{2}}{S_{u}}}\right\}$

$\operatorname{Re}_{v}=\frac{\rho_{u} u_{v}\left(D_{v} / 2\right)}{\mu_{u}}$

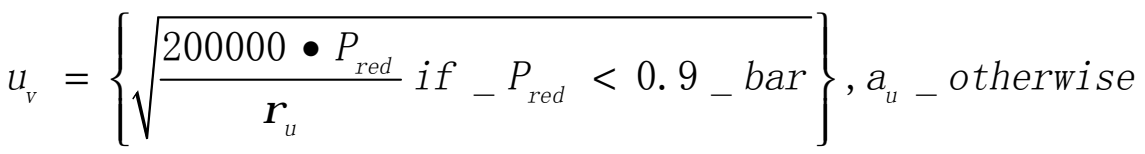

$\mu_{u}$ :the unburned gas-air mixture dynamic viscosity.

$D_{h e}$ :the enclosure hydraulic equivalent diameter.

$D_{v}:$ the vent diameter as determined through iterative calculation.

$\theta=0.39$

$\beta_{1}=1.23$

$\beta_{2}=0.0487$

\section{Effect of transmission device on flame propagation velocity}

When flame propagate in the pipe,the dramatic effect on its propagation is caused by the length,diameter and internal structure of pipe.As gas energy most propagation in cylindrical pipe, the longer the length of pipe is, the longer development time flame has and the final velocity and pressure 
will be higher.However,due to the inner wall is relatively smooth, most of the flame propagation in the pipe will gradually stabilized and DDT won't happen.But if there is any obstacles in pipe,the flame will get accelerate violently.For the influence of obstacles on flame propagation,many scholars have conducted lots of researches[7,8,9]. After summarizing a lot of experimental data, the velocity of flame propagation with an obstacle in the arrangement of the pipe is given in Fig.1.Due to the influence of the obstacles, the velocity of flame have been accelerated a lot and the enhance rate is closely related to the blockage ratio.

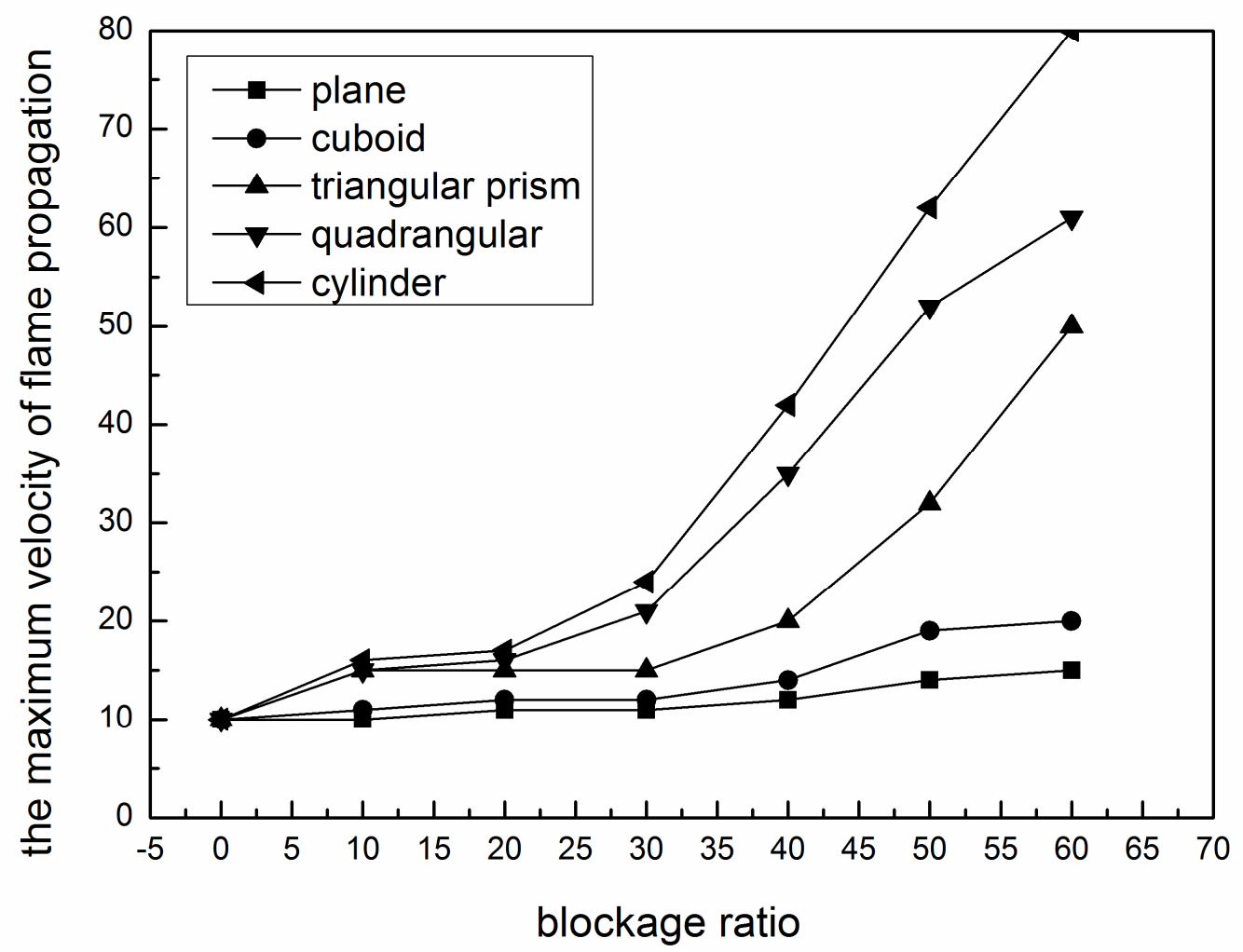

Figure 1 flame velocity varies with blackage ratio

Due to the surface viscous effects of obstacle,velocity gradient and pressure difference will generated.When the flame pass over the obstacles,unburned gas in the front of flame will generate a region in which flame flow around obstacles because of pressure gradient and aggravating the disturbance of flame front.The flame get into the flow field and variety along the gradient,wrinkles appear on the front and make the reactoin area increase. So the flame velocity increase rapidly[10].Combustion from laminar to turbulent may cause the conversion from deflagration to detonation.Therefore it's necessary to keep the inner wall smooth and as far as possible to avoid obstacle appeared in pipeline to ensure the security of transmission.

\section{Conclusions}

1.Gas energy have got large-scale application for the advantages such as clean,safe, and convenient.The security in gas energy transmission now have become the hot issue of corcern.

2. The velocity of flame propagation in pipeline has a direct impact on the pressure and destruction effects,so velocity has become an important factor in the assessment of security.

3. Gas concentration, gas type,temperature,pipe length,internal setting of pipe and other factors will all affect the propagation velocity,we can make security protection in this aspects to reduce accidents. 


\section{References}

[1]Martstein G H.Experimental and theoretical studies of flame front stability[J]. J.Aero.Sci,1951,18:199-209.

[2]Geoff Searby.Acoustic instability in premixed flames[J].Combustion Science and Technology,1992,81:221-231.

[3]Lee S T,Tsai C H.Numerical investigation of steady laminar flame propagation in a circular tube[J].Combustion and flame,1994,99(3):484-9490.

[4]Hengyang Zhao,Principle of gas and dust explosion[M],Beijing Institute of Technology Press, BeiJing, 1996.

[5]Zhichong Gao. The temperature and pressure of $\mathrm{CH}_{4}$ explosive reaction system[J].Journal of LiaoNing University,2008,35(2):143-146.

[6]R.Rota,P.Canu,S.Carra.Vented gas deflagration modeling:A simplified approach[J].Combustion and flame,1991,85:319-330.

[7]I O Moen,M Donato,R Knystautas,et a1.Flame acceleration due to turbulence produced by obstacles[J].Combustion and Fla-me,1980,39(1):21-32.

[8]M Fairweather,G K Hargrave,S S Ibra-him,et al.Studies of premixed flame propagation in explosion tubes[J].Combustion and Flame,1999,116(4):504-518.

[9]Ritsu,Dobash.Experimental study on gas explosion behavior in enclosure[J].Journal of Loss Prevention in the Process Indus-tries,1997,10(2):83-89.

[10]Baiquan Lin, Rengui Zhang,Henghong Lv.Research on Acceleration Mechanism and Flame Transmission in Gas Explosion[J]. Journal of China Coal Society,1999(01):56-59. 